\title{
Anti-transferrin receptor-modified amphotericin B-loaded PLA-PEG nanoparticles cure Candidal meningitis and reduce drug toxicity
}

This article was published in the following Dove Press journal:

International Journal of Nanomedicine

5 October 2015

Number of times this article has been viewed

\author{
Xiaolong Tang, 1,2,* Yong Liang,,* \\ Yongqiang Zhu, ${ }^{1,4, *}$ Chunmei Xie, ${ }^{5}$ \\ Aixia Yao,' Li Chen,' Qinglin \\ Jiang,' Tingting Liu,' Xiaoyu \\ Wang,' Yunyun Qian,' Jia Wei,' \\ Wenxuan Ni,' Jingjing Dai,' \\ Zhenyou Jiang, ${ }^{6,7, *}$ Wei Hou ${ }^{2, *}$ \\ 'Clinical Laboratory, Huainan First People's \\ Hospital and First Affiliated Hospital \\ of Medical College, Anhui University of \\ Science \& Technology, Huainan, ${ }^{2}$ State \\ Key Laboratory of Virology/Institute of \\ Medical Virology, School of Basic Medical \\ Sciences, Wuhan University, Wuhan, ${ }^{3}$ Clinical \\ Laboratory, Huai'an Hospital Affiliated \\ of Xuzhou Medical College, Huaian, \\ ${ }^{4}$ Department of Medical Genetics, Tongji \\ Medical College, Huazhong University of \\ Science and Technology, Wuhan, ${ }^{5}$ School \\ of Biotechnology, Southern Medical \\ University, ${ }^{6}$ Department of Microbiology \\ and Immunology, School of Medicine, \\ ${ }^{7}$ Guangdong Key Laboratory of Molecular \\ Immunology and Antibody Engineering, \\ Jinan University, Guangzhou, People's \\ Republic of China \\ *These authors contributed equally \\ to this work
}

\begin{abstract}
Fatal fungal infections in central nervous system (CNS) can occur through hematogenous spread or direct extension. At present, hydrophobic amphotericin B (AMB) is the most effective antifungal drug in clinical trials. However, AMB is hydrophobic and therefore penetrates poorly into the CNS, and therapeutic levels of AMB are hard to achieve. The transferrin receptor (TfR/CD71) located at the blood-brain barrier mediates transferrin transcytosis. In order to enhance the receptor-mediated delivery of AMB into CNS with therapeutic level, an anti-TfR antibody (OX26)-modified AMB-loaded PLA (poly[lactic acid])-PEG (polyethylene glycol)-based micellar drug delivery system was constructed. The prepared OX26-modified AMB-loaded nanoparticles (OX26-AMB-NPs) showed significant reduction of CNS fungal burden and an increase of mouse survival time. In conclusion, OX26-AMB-NPs represent a promising novel drug delivery system for intracerebral fungal infection.
\end{abstract}

Keywords: drug delivery, nanocarrier, amphotericin B, blood-brain barrier, transferrin receptor

\section{Introduction}

The incidence of intracranial fungal infection is associated with increased prescription of antibiotics and immunosuppressive drugs. Immunosuppression is usually followed by immunodeficiency disease, serious infection, or organ transplantation. ${ }^{1-3}$ However, it is difficult to achieve an effective concentration of antifungal drugs at the site of infection due to the blockade presented by the blood-brain barrier (BBB). ${ }^{4,5}$ Amphotericin $\mathrm{B}$ (AMB) is the broadest spectrum systemic antifungal agent available, but is no longer the primary drug of choice for invasive fungal infection due to its inherent low solubility, poor efficacy, and nephrotoxicity. ${ }^{6}$ These adverse effects often lead to treatment interruptions because even modest doses can cause renal damage. In order to improve the therapeutic index of AMB, reduce its associated toxicity, and increase solubility, newer formulations of AMB that do not cause impairment in renal function are urgently needed.

Formulations of AMB, such as nanoparticles (NPs) and liposomes, have been designed to enhance permeability across the BBB. ${ }^{7}$ AMB colloidal dispersion, an injected AMB cholesteryl sulfate complex, was developed to reduce toxicity while reducing the concentration needed for tissue distribution of the drug without limiting antifungal efficacy. ${ }^{8,9}$ Synthetic biodegradable copolymer hydrophobic poly(lactic acid) (PLA) has also been trialed as an AMB delivery material for its capacity to protect drugs against degradation while mediating sustained release. ${ }^{10,11}$ However, PLA is hydrophobic, has a low drug loading capacity for polar drugs, a long degradation time, ${ }^{12}$ and these PLA NPs can be easily captured by the reticuloendothelial system
Correspondence: Zhenyou Jiang Guangdong Key Laboratory of Molecular Immunology and Antibody Engineering, Jinan University, Guangzhou 510632, People's Republic of China

Email tjzhy@jnu.edu.cn

Wei Hou

State Key Laboratory of Virology/lnstitute of Medical Virology, School of Basic Medical Sciences, Wuhan University, Wuhan 43007I,

People's Republic of China

Email houwei@whu.edu.cn 
(RES) in vivo, ${ }^{13}$ which in turn prevents this delivery vehicle from reaching their designated target site. Additional excipients such as polyethylene glycol (PEG) has many advantages such as antiphagocytosis against macrophages, good hydrophilicity, and biocompatibility. ${ }^{14}$ Moreover, PEG can also effectively mitigate RES-mediated particle clearance, which protects the particle from recognition by the RES. ${ }^{15}$ PLA copolymerization with PEG can improve hydrophilicity, reduce the burst effect, increase the drug loading, and prolong the in vivo residence time of drugs, showing great potential in development for drug delivery.

To overcome the shortcomings of PLA, amphiphilic PEG polymer-block-PLA, such as diblock polymer of PLAPEG which can self-assemble into nanomicelles and entrap medicines simultaneously and improve the loading rate of medicine compared with microscale formulations, avoiding or minimizing the use of surfactants and organic solvents has been applied for the encapsulation of medications with small molecular weight. ${ }^{16}$ Therefore, PLA-PEG encapsulation may represent a model of hydrophobic drug delivery system. However, the clinical efficacy of PLA-PEG for the treatment of intracranial infection was restricted by poor permeability across the BBB. Fortunately, this therapeutic delivery system could be modified to allow rapid passage through the $\mathrm{BBB}$ to the brain via endogenous $\mathrm{BBB}$ transport systems. ${ }^{16,17}$

The transferrin receptor (TfR), one component of the BBB, is abundantly expressed on the brain capillary endothelium. ${ }^{17-19}$ A rat TfR monoclonal antibody (mAb), OX26, is proven to be able to bind to an extracellular domain of TfR and is transferred into the BBB via the endogenous transferrin transport system. ${ }^{17}$ Under this mechanism, OX26 could enhance transport of conjugated drugs across the BBB. ${ }^{18,19}$ For that reason, we attempted to harness OX26 to transfer the AMB-loaded nanodrugs through the BBB and construct a brain-targeted drug delivery system.

However, the antifungal efficacy, clinical efficacy, and toxicity of this OX26-modified AMB-loaded PLA-TPGS (D- $\alpha$-tocopheryl polyethylene glycol 1000 succinate) block copolymer remained to be determined. In this work, we fabricated PLA-TPGS NPs by combining the two amphiphilic components, PLA and TPGS, and evaluated the antifugal efficacy, stability, antifungal mechanism, and toxicity of the AMB-loaded NPs in vitro and in vivo. It is demonstrated that the drug NPs exert improved antifugal efficacy and lower toxicity than free AMB. Our NPs minimized the delivery of AMB to host cells and maximized the accumulation in fungal cells, indicating that amphiphilic self-aggregated NPs act as a hydrophobic drug carrier for delivery of AMB.

\section{Materials and methods Materials}

MTT [3-(4,5-dimethylthiazol-2-yl)-2,5-diphenyltetrazolium bromide], L-lactide, polyethylene glycol diamine (PEG$\mathrm{NH}_{2}$; molecular weight $\left.3.5 \mathrm{kDa}\right)$, hydrazine, ethyl-3-(3dimethylaminopropyl)carbodiimide hydrochloride (EDC), stannous octoate [Sn (Oct) 2: stannous 2-ethylhexanoate], $\mathrm{N}$-hydroxysuccinimide (NHS), dicyclohexylcarbodiimide, and dimethyl sulfoxide (DMSO) were obtained from Sigma (St Louis, MO, USA). PLA-PEG diblock (15\% PEG with $5 \mathrm{kDa}$ ) was obtained from Boehringer Ingelheim (Ingelheim am Rhein, Germany). All the other chemicals and reagents used were of analytical purity grade or higher and were obtained commercially. AMB was purchased from Sinopharm (Shanghai, People's Republic of China) as an analytical grade preparation. RPMI (Roswell Park Memorial Institute) 1640 medium and penicillin/streptomycin solution were obtained from Gibco Invitrogen (Carlsbad, CA, USA). 4',6'-Diamidino-2-phenylindole (DAPI) was purchased from Molecular Probes (Eugene, OR, USA). Anti-TfR antibody (OX26) was obtained from Abcam (Cambridge, UK).

\section{Preparation of OX26-modified AMB-loaded amphiphilic PLA-PEG block copolymer nanoparticles}

Preparation of the PLA-PEG block copolymer containing the terminal $\mathrm{NH}_{2}$ group began with the synthesis of monocarboxylated PEG as previously described by Tang et al. ${ }^{18}$ Briefly, vacuum-dried lactide $(16.0 \mathrm{~g})$ and PEG- $\mathrm{NH}_{2}(3.0 \mathrm{~g})$ were allowed to react in anhydrous toluene in the presence of the catalyst tin(II) 2-ethylhexanoate (200 $\mathrm{mg}$ ) at the refluxing temperature of toluene. After evaporation of the reaction solvent, the PLA-PEG copolymer was extracted in chloroform. Prepared PLA-PEG-NH $(1.0 \mathrm{~g})$ was activated by EDC $(50.0 \mathrm{mg})$ and NHS $(40.0 \mathrm{mg})$ in DMSO $(10 \mathrm{~mL})$ for 5 hours at room temperature. To prepare the OX26-functionalized copolymer, the prepared OX26 was added to the activated copolymer in DMSO and allowed to react for an additional 48 hours. The mixture was then dialyzed against deionized water to remove unreacted OX26. The conjugation of OX26 to the copolymer was confirmed by ${ }^{1} \mathrm{H}$ NMR (proton nuclear magnetic resonance) spectroscopy.

To synthesize the OX26-AMB-NPs, a preweighed amount of AMB and $100 \mathrm{mg}$ of PLA-PEG-OX26 copolymer were dissolved in $8 \mathrm{~mL}$ of acetone by vortexing and sonication. The resulting NP suspension was then stirred at room temperature overnight to completely remove acetone. The NP suspension was centrifuged at $80,000 \times g$ for 15 minutes before washing 2-3 times to remove the emulsifier and 
unloaded drug. The dispersion was then lyophilized for 48 hours (Figure 1). Coumarin 6-loaded PLA-PEG-OX26 NPs (OX26-coumarin 6-NPs), AMB-loaded PLA-PEG NPs (AMB NPs), and coumarin 6-loaded PLA-PEG NPs (coumarin 6-NPs) were fabricated in a similar procedure. Scanning electron microscopy images of these NPs were obtained with a JEOL JSM6700F electron microscope (JEOL, Tokyo, Japan). Dynamic light scattering for size determination and $\zeta$ potential measurements was performed on a Malvern Zetasizer Nano ZS90 instrument (Malvern Instruments, Malvern, UK). The particle size distribution was determined by Malvern Zetasizer 2000 (Malvern Instruments). Morphology of the NPs was examined using variable pressure scanning electron microscopy (Hitachi 3400N; Hitachi, Gaithersburg, MD, USA). The zeta potential of particles was measured using a Malvern Zetasizer 2000 (Malvern Instruments). The drug (AMB)-entrapment efficiency (EE\%) of the NPs was measured by high-performance liquid chromatography (HPLC) (LC 1200; Agilent Technologies, Santa Clara, CA, USA) and calculated with the following equation:

$$
\mathrm{EE} \%=\frac{\text { Amount of AMB in NPs }}{\text { Total amount of feeding AMB }} \times 100 \%
$$

In vitro release of $\mathrm{AMB}$ from the $\mathrm{AMB}$ formulations in phosphate-buffered saline (PBS), serum, and organs was evaluated by HPLC by following an established method..$^{20} \mathrm{AMB}$ was extracted from the serum by addition of two parts methanol to one part plasma and vortexed for 15 minutes. The serum-methanol mixtures were centrifuged at $6,000 \times g$ for 15 minutes. Different organs of the experimental mice were homogenized in tridistilled water. AMB was extracted from the homogenate by addition of two parts methanol to one part homogenate. The supernatants were filtered using a microsyringe filter $(0.22 \mu \mathrm{m}$ polyethersulfone membrane; Millipore, Billerica, MA, USA) after centrifugation at $6,000 \times g$ for 30 minutes.

HPLC was performed with eluent ethylenediaminetetraacetic acid (20.0 $\mathrm{mM}$ in tridistilled water) and acetonitrile $(60: 40 \mathrm{v} / \mathrm{v})$ at a flow rate of $1.2 \mathrm{~mL} / \mathrm{min}$, with retention time for AMB of 11 minutes and for the internal standard of 17 minutes, injection volume of $100 \mu \mathrm{L}$, and detection at $405 \mathrm{~nm}$. The calibration curve was obtained using mixtures of AMB and internal standard $(10 \mu \mathrm{g} / \mathrm{mL})$ by least squares linear regression analysis. The peak area ratio of AMB to internal standard versus nominal concentration of the drug was plotted.

A
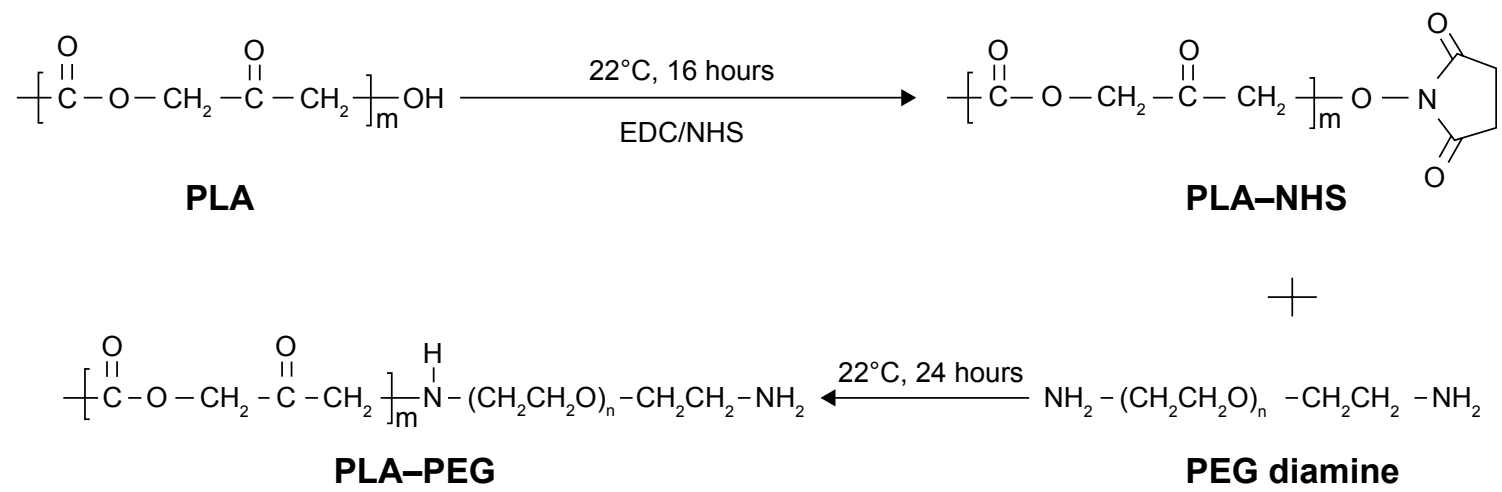

B

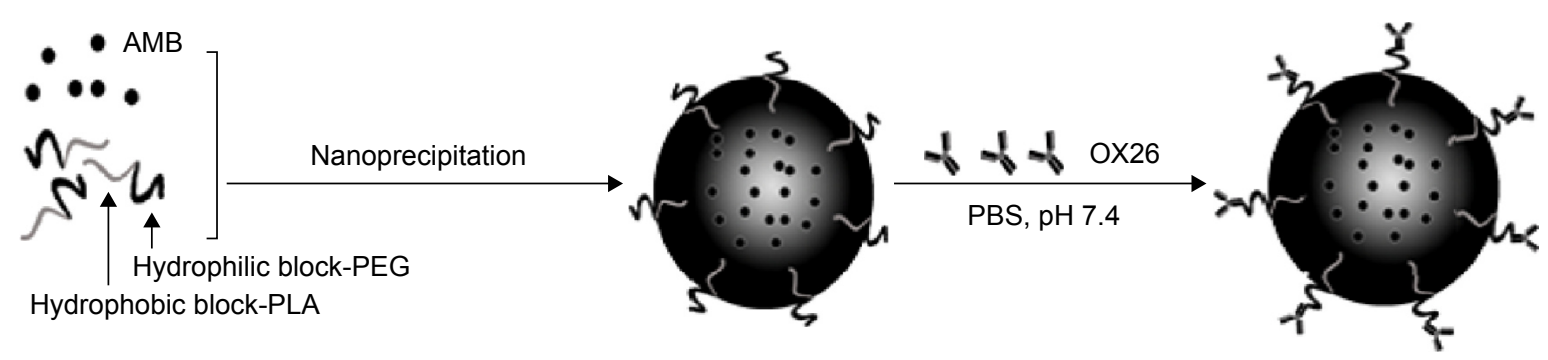

Figure I Illustration of PLA-PEG copolymer synthesis (A) and OX26-modified AMB-loaded NPs synthesis (B).

Abbreviations: AMB, amphotericin B; EDC, ethyl-3-(3-dimethylaminopropyl) carbodiimide hydrochloride; NHS, N-hydroxysuccinimide; NP, nanoparticle; OX26, TfR monoclonal antibody of rats; PBS, phosphate-buffered saline; PEG, poly(ethylene glycol); PLA, poly(D,L-lactide); TfR, transferrin receptor. 


\section{Drug release assay}

The in vitro release of AMB from AMB NPs was determined by measuring the amount of residual AMB in the NPs. ${ }^{12}$ Briefly, $5 \mathrm{mg}$ of lyophilized AMB NPs were transferred into a centrifuge tube and redispersed in $8 \mathrm{~mL}$ of PBS (pH 7.4) containing $0.1 \% \mathrm{w} / \mathrm{v}$ Tween 80 . The tube was rotated at $135 \mathrm{rpm}$ at $37^{\circ} \mathrm{C}$. At certain time intervals, the tube was centrifuged at $80,000 \times g$ for 15 minutes. The supernatant was then transferred into a glass test tube for HPLC. The pellet was resuspended in $8 \mathrm{~mL}$ of fresh PBS for subsequent analysis. The cumulative release amount of AMB from NPs was plotted against time.

\section{Toxicity of AMB-loaded NPs}

Many studies have shown that PLA-PEG NPs have few adverse effects, ${ }^{19-23}$ while AMB can cause hemolysis and kidney damage by binding membrane lipids. ${ }^{21}$ Therefore, we mainly evaluated the effect of AMB NPs and OX26-AMB-NPs on blood cell hemolysis and on renal cell toxicity. Drug-induced liver injury was also evaluated by hepatic histopathology, cellular apoptosis using molecular terminal deoxynucleotidyl transferase-mediated dUTP-biotin nick end labeling (TUNEL) assay, and serological indexes of liver damage ( $\alpha$-glutathione$S$-transferase $[\alpha-\mathrm{GST}]$ and alanine aminotransferase [ALT], glutamate dehydrogenase [GLDH]).

The hemolytic activity of free AMB and AMB-loaded nanospheres was determined as previously described. ${ }^{12}$ Briefly, erythrocytes from the heparin anticoagulant blood of five 6-week-old, specific-pathogen-free New Zealand White rabbits were collected by centrifugation $(1,200 \times g$ for 15 minutes) and washed three times in PBS. The animal experiment was approved by the Committee of Laboratory Animal Welfare and Ethics, Anhui University of Science and Technology.

To study the extent of hemolysis, $0.2 \mathrm{~mL}$ of erythrocytes $\left(1.0 \times 10^{7}\right.$ cells $\left./ \mathrm{mL}\right)$ was incubated with $0.2 \mathrm{~mL}$ of the various AMB formulations (containing 0.5, 1.0, 2.0, 4.0, 8.0, 16.0, $32.0,64.0,128.0$, and $256.0 \mu \mathrm{g} / \mathrm{mL}$ AMB equivalents) at $37^{\circ} \mathrm{C}$ for 72 hours. The free form of AMB was dissolved in $20 \mu \mathrm{L}$ of DMSO and made up to $0.4 \mathrm{~mL}$ with PBS. After the stipulated periods of time, the reaction mixture was centrifuged at $800 \times g$ for 10 minutes. The supernatant was then collected and analyzed by ultraviolet-visible spectroscopy $\left(\lambda_{\max }=540\right.$ $\mathrm{nm}$ ) for released hemoglobin. The erythrocyte suspension that was dispersed in deionized water, considered to be producing $100 \%$ hemolysis, was used as the positive control and the erythrocyte suspension in PBS served as the negative control. The release of hemoglobin into the supernatant (the hemolysis rate, $\mathrm{HE} \%$ ) was analyzed by measuring absorbance at optical density $(\mathrm{OD})_{540 \mathrm{~nm}}$ with a multiwell microplate reader and was calculated by the following equation:

$$
\mathrm{HE} \%=\frac{\mathrm{OD}_{\text {mean of various } \mathrm{AMB} \text { formulations }}-\mathrm{OD}_{\text {mean of negative control }}}{\mathrm{OD}_{\text {mean of positive control }}-\mathrm{OD}_{\text {mean of negative control }}} \times 100 \%
$$

The cellular toxicity of free AMB, AMB NPs, and OX26AMB-NPs was determined by culturing each molecule with the human embryonic kidney cell line CCC-HEK-1. Cells were suspended at $1 \times 10^{6} / \mathrm{mL}$ and serial dilutions of the drugs were added in triplicate. A $2 \%(\mathrm{v} / \mathrm{v})$ solution of Triton $\mathrm{X}-100$ was used as the $100 \%$ cell lysis positive control. The plates were incubated at $37^{\circ} \mathrm{C}, 5 \% \mathrm{CO}_{2}$ for 3 days, after which MTT was added and cell viability determined at OD at $570 \mathrm{~nm}$. The AMB concentration required to kill $50 \%$ of the cells ( $50 \%$ cytotoxic concentration) was calculated using sigmoidal regression analysis by plotting a graph of the ODs against the AMB concentration and by taking the OD of the control well as $100 \%$ viability. ${ }^{22}$

\section{OX26-AMB-NPs targeting to TfR on cells}

In order to analyze the specificity of OX26-AMB-NPs to TfR on cells, coumarin-6 served as a model control molecule, which can be entrapped in OX26-PLA-PEG NPs, uptaken by $\mathrm{TfR}^{+}$cells followed by quantification. Two tumor cell lines (HeLa and SKOV3) that overexpress TfR on the cell surface and a transformed normal human cell line (HEK 293, TfR ${ }^{\text {low }}$ ) were incubated with the TfR-targeted NPs (OX26-AMB-NPs) at a particle concentration of $50 \mu \mathrm{g} / \mathrm{mL}$ at $37^{\circ} \mathrm{C}$. All cell experiments were carried out with the approval of the Ethics Committee of the Anhui University of Science and Technology. After 4 hours, cells were fixed by acetone for 10 minutes before being stained with DAPI for 20 minutes to highlight the nuclei. After rinsing three times with PBS, cells were observed by confocal laser scanning microscopy (LSM 410, Zeiss, Jena, Germany) with imaging software (Zeiss LSM Image browser, Carl Zeiss Meditec AG, Jena, Germany).

\section{Animal experiments}

\section{Fungal meningitis model establishment}

A murine model of fungal meningitis was established as previously described. ${ }^{23}$ In brief, BALB/c mice weighing 25-30 g were purchased from the Institute of Laboratory Animal Sciences at the Chinese Academy of Medical Science. All animal experiments were carried out with the approval of the Ethics Committee of the Anhui University of Science and Technology. On the day of Candida glabrata inoculation (day 0), 
$\sim 10^{5}$ conidia in $20 \mu \mathrm{L}$ PBS was inoculated intracerebrally. Mice were observed until they fully recovered from inhaled anesthesia and had no sign of neurological abnormalities, which took 10 minutes.

\section{Experimental treatment}

\section{Drug serum and brain distribution analysis}

All mice were assigned randomly AMB group, AMB NPs group, and OX26-AMB-NPs group. AMB, AMB NPs, and OX26-AMB-NPs were reconstituted according to the manufacturer's instructions and diluted with sterile $5 \%$ dextrose on the day of administration. Each group included 12 mice. AMB or other AMB formulations were administered once with AMB $3.0 \mathrm{mg} / \mathrm{kg}$. All drugs were administrated by slow intravenous push via the lateral tail vein. Every 12 hours post the administration, two mice from each group were randomly selected and sacrificed during the following 72 hours, and $0.3 \mathrm{~mL}$ of their blood was centrifuged at $2,800 \times g$ for 20 minutes. The supernatant was collected and stored at $-80^{\circ} \mathrm{C}$ until determination. Two grams of brain tissue was homogenized in one volume of sterile saline, and then placed in a preweighed Eppendorf tube and stored at $-80^{\circ} \mathrm{C}$ until further processing. The AMB concentration in serum or brain tissues was determined by HPLC.

\section{Drug effectiveness evaluation}

All mice were assigned randomly to PBS control group, null NPs group, AMB group, AMB NPs group, and
OX26-AMB-NPs group. Each group included 20 mice. AMB, AMB NPs, and OX26-AMB-NPs were reconstituted according to the manufacturer's instructions and diluted with sterile $5 \%$ dextrose on the day of administration. AMB or other AMB formulations were administered on day 3 after C. glabrata inoculation and for 3 consecutive days (two times per day for 3 consecutive days, with AMB $3.0 \mathrm{mg} /$ $\mathrm{kg} / \mathrm{d}$ ) to maintain a stable blood drug concentration, as the serum half-life of unmodified AMB is approximately 24 hours, which is demonstrated by the drug serum and brain distribution analysis (Figure 2A).

Drug efficacy was determined by measuring the survival rate, evaluating the brain fungal burden, and histopathologic examination. ${ }^{23,24}$ To record, survival cages were inspected twice daily for 24 days postinfection. For histopathologic examination, three mice from each group were killed on day 15 postinfection, and brain tissues were preserved with $4 \%$ buffered formalin for histopathologic analysis. Brain slices were stained with HE and Gram's/Brilliant green stain to evaluate histopathological changes.

\section{Toxicity assessment}

Three days after treatment, two mice from each group were sacrificed, and a kidney and other major organs were fixed with formaldehyde. For light microscopic evaluation, portions of kidney specimens from each group were cut into $5 \mu \mathrm{m}$ thick sections and stained with HE or immunohistochemical
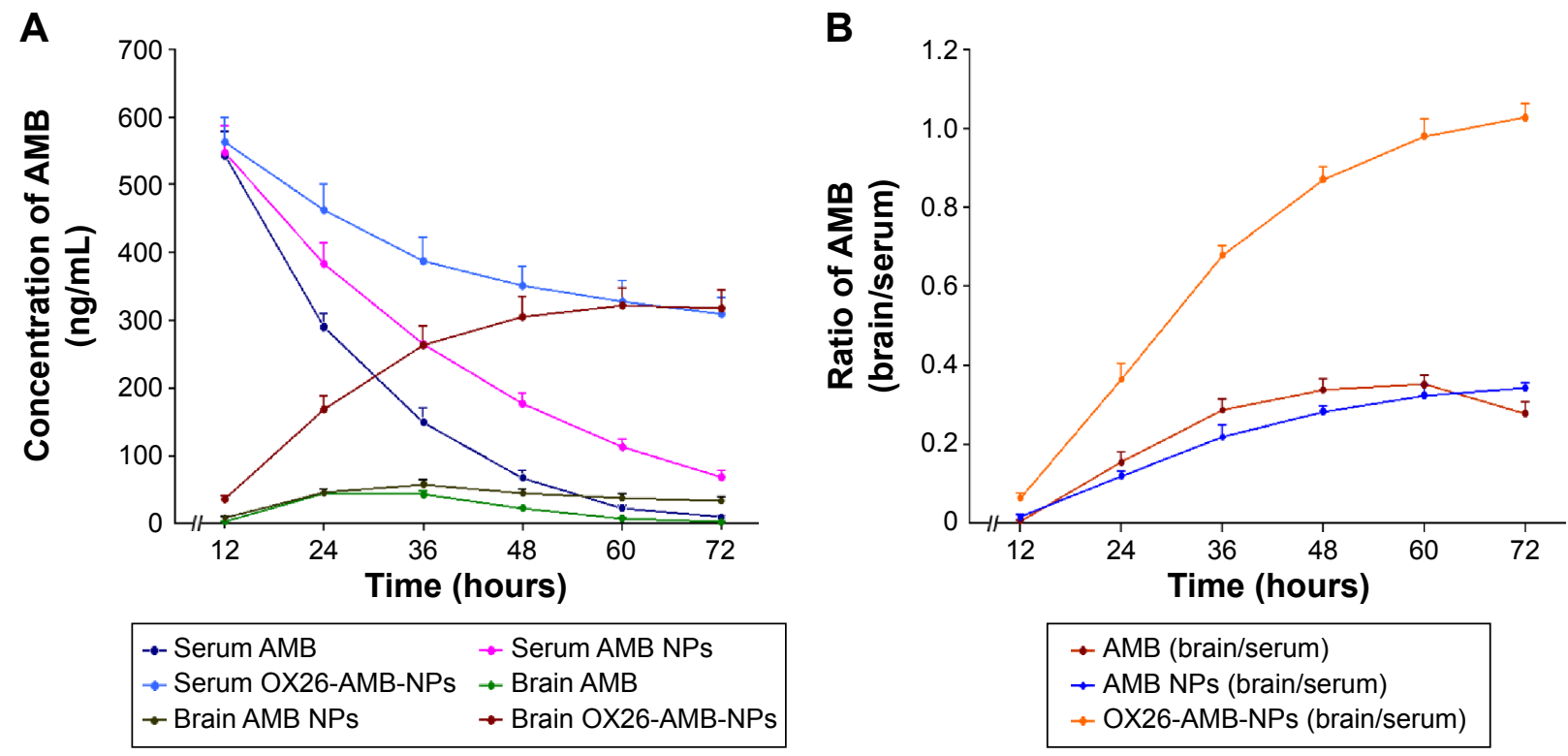

Figure 2 On day 6 after inoculation, (A) the levels of AMB in serum and brain were measured by HPLC, (B) shows the ratio of concentrations of AMB in brain to serum. Notes: Each group included five animals. Data are expressed as the mean \pm SEM.

Abbreviations: AMB, amphotericin B; HPLC, high-performance liquid chromatography; NP, nanoparticles; OX26, TfR monoclonal antibody of rats; SEM, standard error of mean; TfR, transferrin receptor. 
(IHC) stains. Kidney injury molecule-1 (KIM-1) and neutrophil gelatinase-associated lipocalin (NGAL) were used to evaluate the glomerular and tubular epithelial alterations (injury, inflammation, degeneration, apoptosis, and necrosis). ${ }^{24}$ To assess early damage caused by exposure to AMB, cellular apoptosis was measured using TUNEL assay. As $\alpha$-GST, ALT and GLDH are effective and sensitive serological indexes of liver damage, these proteins represent indicators of the degree of liver injury. ${ }^{25}$ Therefore, we assessed liver damage by measuring histopathological changes, the activity of serum enzyme markers ALT and GLDH, determined by standard clinical automated analyses (Roche, Basel, Switzerland), and serum $\alpha$-GST level measured using an antirat $\alpha$-GST enzyme immunoassay (Hepkit alpha GST; Biotrin, Dublin, Ireland). Histopathological changes in the lung, heart, and brain and measured apoptosis were also evaluated by TUNEL assay using the FragEL DNA fragmentation detection kit (QIA33; Calbiochem, La Jolla, CA, USA).

\section{Statistical analysis}

Qualitative data were expressed as mean \pm standard error of mean. Differences between two groups were analyzed by Student's $t$-test. A value of $P<0.05$ was considered statistically significant. Statistical analysis was performed with SPSS version 11.0 for Windows (SPSS Inc., Chicago, IL, USA).

\section{Results}

\section{NP characteristics}

\section{The physical characteristics of PLA-PEG NPs}

PLA-PEG NPs were prepared using the solvent evaporation method. Figure 3A shows the morphology of AMB NPs and OX26-AMB-NPs observed by transmission electron microscope. The OX26-AMB-NPs exhibited a rigid core with a fuzzy appearance around the surface, representing the antibody OX26 around the PLA-PEG NP surface. Covering NP surfaces with hydrophilic PEG has been reported to stabilize
A
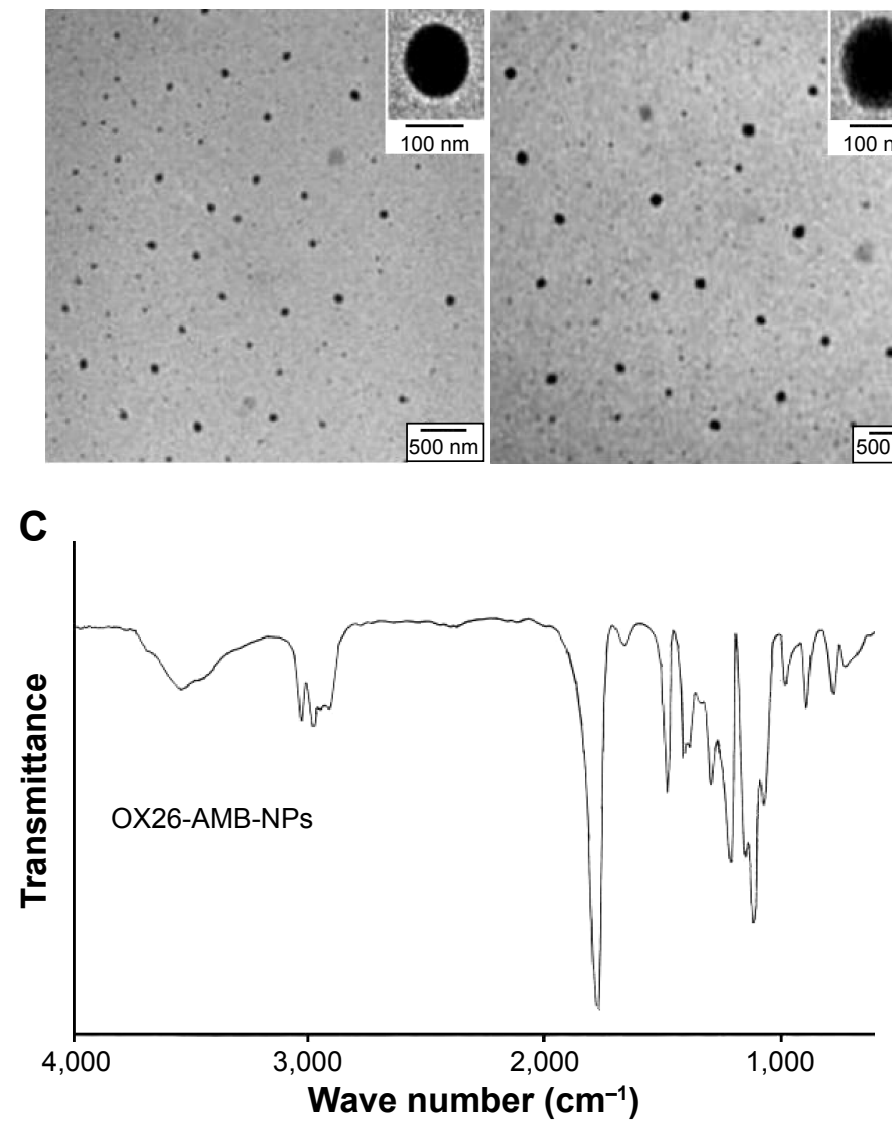

B
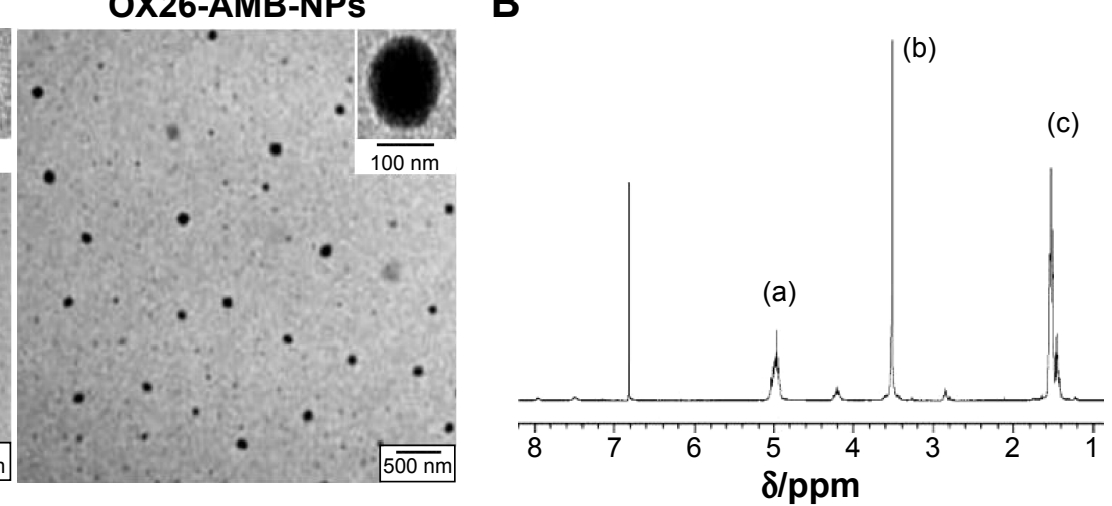

D Size distribution by intensity

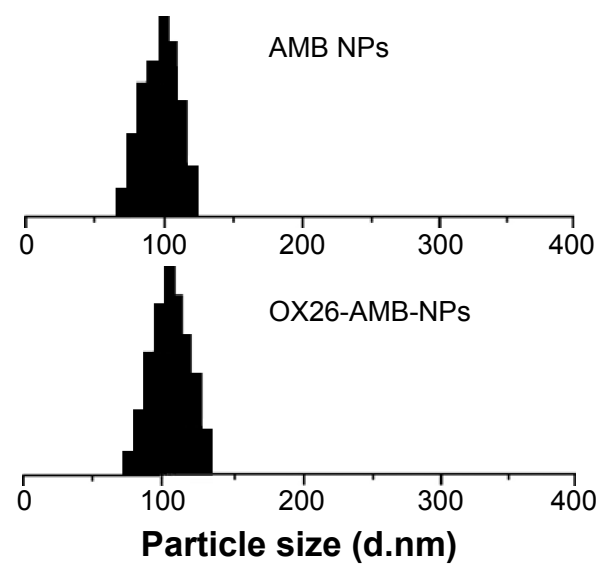

Figure 3 Characterization of PLA-PEG copolymer.

Notes: (A) TEM images of AMB NPs and OX26-AMB-NPs. (B) 'H NMR spectrum of PLA-PEG (500 MHz, CDCl $)$ ). The characteristic peaks of PLA ([a] CH:5.2 Ppm and [c] CH3:I.6 PPm) and PEG ([b] CH2:3.6 Ppm). (C) FTIR spectrum of PLA-PEG. Approximately $0.20 \mathrm{mg}$ of the dry sample was mixed with IR-grade KBr (0.12 g) and pressed (10 ton) into tablet form. (D) DLS analysis of OX26-AMB-NPs and AMB NPs in water $(0.020 \mathrm{mg} / \mathrm{mL})$.

Abbreviations: AMB, amphotericin B; DLS, dynamic light scattering; FTIR, Fourier transform infrared spectroscopy; 'H NMR, proton nuclear magnetic resonance; IR, infrared; NP, nanoparticle; OX26, TfR monoclonal antibody of rats; PEG, polyethylene glycol; PLA, poly(lactic acid); TEM, transmission electron microscope; TfR, transferrin receptor. 
NP against opsonization and subsequent phagocytosis, and thus prolongs the circulation time of NPs in vivo. ${ }^{12}$

The ${ }^{1} \mathrm{H}$ NMR spectrum of PLA-PEG copolymer (Figure 3B) was used to estimate the average molecular weight $\left(M_{w}\right)$ of the copolymer by comparing the peak ratio of the methylene protons of PEG $\left(-\mathrm{OCH}_{2} \mathrm{CH}_{2}-: \delta 3.6 \mathrm{ppm}\right)$ with the peak ratio of methine protons of PLA $(\mathrm{CH}: \delta 5.2 \mathrm{ppm})$. The $M_{\mathrm{w}}$ of PEG was $4,000 \mathrm{~g} / \mathrm{mol}$ and the weight average $M_{\mathrm{w}}$ of PLA-PEG copolymer was approximately $28.0 \mathrm{kDa}$.

The critical micelle concentration of the amphiphilic PLA-PEG copolymer was determined by using pyrene as a fluorescence probe. No micelles were formed at a concentration below the critical micelle concentration of $5.2 \times 10^{-7} \mathrm{~mol} / \mathrm{L}$, which was close to the optimum concentration $\left(8.0 \times 10^{-7} \mathrm{~mol} / \mathrm{L}\right)$ needed for the micelles to be stable in the bloodstream. Therefore, our PLA-PEG micelles had the potential to retain their core-shell conformation in the circulation.

The structure of monocarboxylated PLA-PEG copolymer was confirmed by the infrared spectrum (Figure $3 \mathrm{C}$ ). The characteristic sharp peak at $1,755 \mathrm{~cm}^{-1}$ is attributed to the carbonyl $(\mathrm{C}=\mathrm{O})$ group in the PLA block of the PLAPEG copolymer. Peaks related to the $\mathrm{C}-\mathrm{O}-\mathrm{C}$ stretching and $\mathrm{C}=\mathrm{O}$ group in $\mathrm{PEG}$ appeared at 1,090 and $1,650 \mathrm{~cm}^{-1}$, respectively.

The infrared spectrum strongly indicated the ring-opening polymerization of lactide and the formation of PLA-PEG block copolymer. The average size and zeta potential of null PLA-PEG, AMB-PLA-PEG, and OX26-AMB-PLA-PEG in deionized water were measured by dynamic light scattering technique (Table 1). Zeta potential of the prepared AMBPLA-PEG and OX26-AMB-PLA-PEG NPs was -21.5 \pm 1.6 and $-18.1 \pm 1.3 \mathrm{mV}$, respectively. Both NPs were negatively charged and were able to avoid nonspecific electrostatic interactions with the cell membranes. The EE\% of PLA-PEG

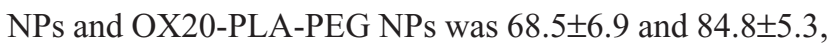
respectively. The mean diameters of the null PLA-PEG, AMB-PLA-PEG, and OX26-AMB-PLA-PEG were 99.5, 113.3, and $121.8 \mathrm{~nm}$, respectively. As shown in Figure 3A and D and Table 1, all of NPs had a spherical shape and uniform size and good dispersion. Polydispersity index values also indicated that these fabricated NPs maintained an ideal homogeneous particle diameter, zeta potential, dispersion, and core-shell structures. ${ }^{26}$

\section{In vitro release of AMB-loaded NPs}

The cumulative release profiles of AMB NPs and OX26AMB-NPs are shown in Figure 4. The release of AMB from AMB NPs exhibited dual phase characteristics in vitro. The initial burst release occurred after the first 48 hours, followed by relatively slow release for up to 7 days before the remaining stage. After 7 days, $\sim 83.2 \% \pm 3.9 \%$ of AMB was released, and after 12 days, AMB NPs were entirely released.

Regarding OX26-AMB-NPs, the release of AMB continued with almost uniform release characteristics. However, compared with the AMB NPs, $\sim 96.1 \% \pm 3.6 \%$ was released within 7 days. OX26-AMB-NPs exhibited approximately uniform stability release.

\section{Drug cytotoxicity}

The hemolytic ability of free AMB and AMB-loaded NPs was measured in vitro (Figure 5). The maximum hemolytic ability (100\% lysis) of AMB, AMB-loaded NPs, and OX26-AMB-NPs was 45.0, 100.0, and $250.0 \mu \mathrm{g} / \mathrm{mL}$, respectively, and null-loaded NPs had no hemolytic ability (lower than $1.0 \%$ lysis of erythrocytes) up to $0.5 \mathrm{mg} / \mathrm{mL}$ (data not shown). As shown in Figure 5A, the concentration of OX26-AMB-NPs required to lyse $50 \%$ of erythrocytes was $160.0 \mu \mathrm{g} / \mathrm{mL}$, much higher than the concentration of free AMB $(\sim 27.0 \mu \mathrm{g} / \mathrm{mL})$ or AMP NPs $(\sim 71.0 \mu \mathrm{g} / \mathrm{mL})$ required.

The cytotoxicity induced by various AMB formulations was measured by MTT assay, which reflects the metabolism of the cells (Figure 5B). On incubation with the human embryonic kidney cell line, CCC-HEK-1, free AMB exhibited the lowest CC50 (4.6 $\pm 0.27 \mu \mathrm{g} / \mathrm{mL})$, followed by AMB NPs $(15.37 \pm 1.8 \mu \mathrm{g} / \mathrm{mL})$, and OX26-AMB-NPs $(45.65 \pm 2.5 \mu \mathrm{g} / \mathrm{mL})$. These results demonstrated that the

Table I The particle size, PDI, zeta potential, and EF\% of the PLA-TPGS copolymer $(n=3)$

\begin{tabular}{lllll}
\hline Polymer & Particle size $(\mathrm{nm})$ & Polydispersity index & Zeta potential $(\mathrm{mV})$ & Encapsulation efficiency $(\%)$ \\
\hline Null PLA-PEG & $99.5 \pm 5.6$ & $0.05 \pm 0.02$ & $-23.4 \pm 2.01$ & \\
AMB-PLA-PEG & $113.3 \pm 8.3$ & $0.06 \pm 0.03$ & $-21.5 \pm 1.6$ & $68.5 \pm 6.9$ \\
OX26-AMB-PLA-PEG & $121.8 \pm 9.9$ & $0.06 \pm 0.03$ & $-18.1 \pm 1.3$ & $84.8 \pm 5.3$ \\
\hline
\end{tabular}

Note: Values are reported as mean $\pm \operatorname{SEM}(n=3)$.

Abbreviations: AMB, amphotericin B; EF\%, encapsulation efficiency in \%; PDI, polydispersity index; OX26, TfR monoclonal antibody of rats; PEG, polyethylene glycol; PLA, poly(lactic acid); SEM, standard error of mean; TfR, transferrin receptor; TPGS, D- $\alpha$-tocopheryl polyethylene glycol I000 succinate. 


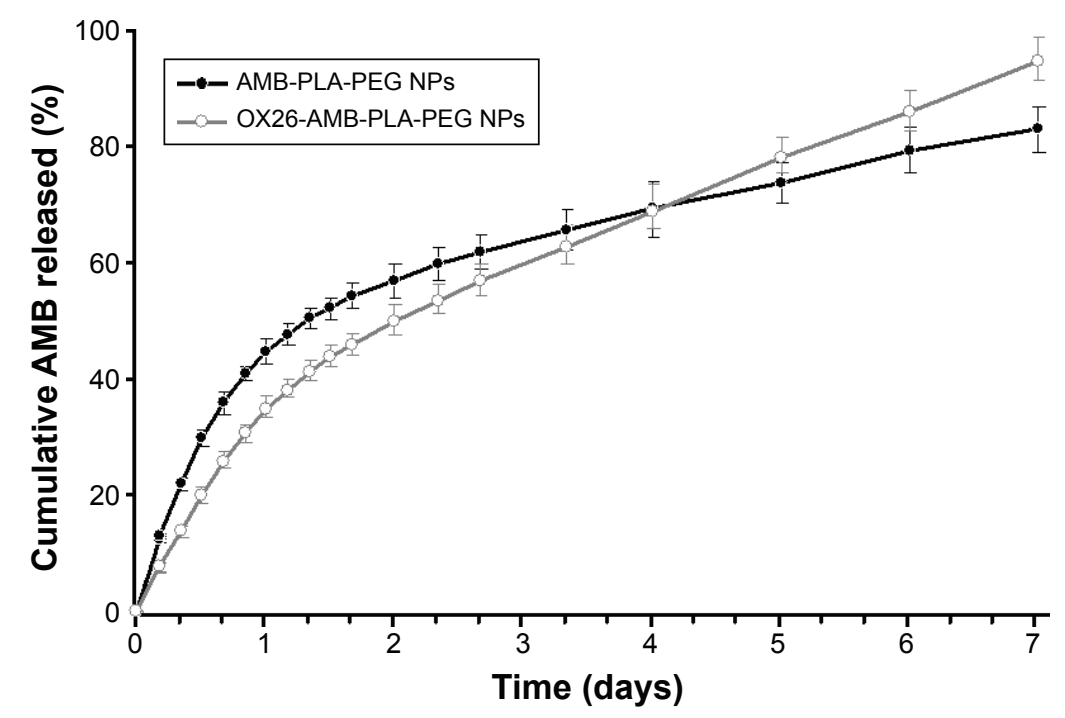

Figure 4 Cumulative release profiles of AMB-NPs and OX26-AMB-NPs in PBS $(\mathrm{pH}=7.35)$ at $37^{\circ} \mathrm{C}$.

Abbreviations: AMB, amphotericin B; NP, nanoparticle; OX26, TfR monoclonal antibody of rats; PBS, phosphate-buffered saline; PEG, polyethylene glycol; PLA, poly(lactic acid); TfR, transferrin receptor.

OX26-AMB-NP formulation was considerably less cytotoxic than free $\mathrm{AMB}$ or AMB NPs.

\section{Nephrotoxicity assessment}

KIM-1, a type I transmembrane glycoprotein that has been reported to be correlated with proximal tubular injury, renal tubular regeneration, and immune response by nephrotoxicants and can be used as a more sensitive biomarker than traditional nephrotoxic biomarkers, like blood urea nitrogen and serum creatinine, and is expressed at high levels in injured kidney. ${ }^{24} \mathrm{NGAL}$ is a $25 \mathrm{kDa}$ protein, which can often be induced in proximal tubule cells by drug-induced nephrotoxicity and is recognized as a sensitive biomarker for the early diagnosis of acute kidney injury. ${ }^{24}$

Staining of paraffin kidney sections treated with $1 \mathrm{mg} / \mathrm{kg}$ of AMB exhibited no obvious nuclear shrinkage or apoptotic marker expression in renal tubular epithelial cells (Figure 6), but high levels of KIM-1 and NGAL expressions were observed. AMB-NP-treated mice exhibited moderate congestion in the medulla and the
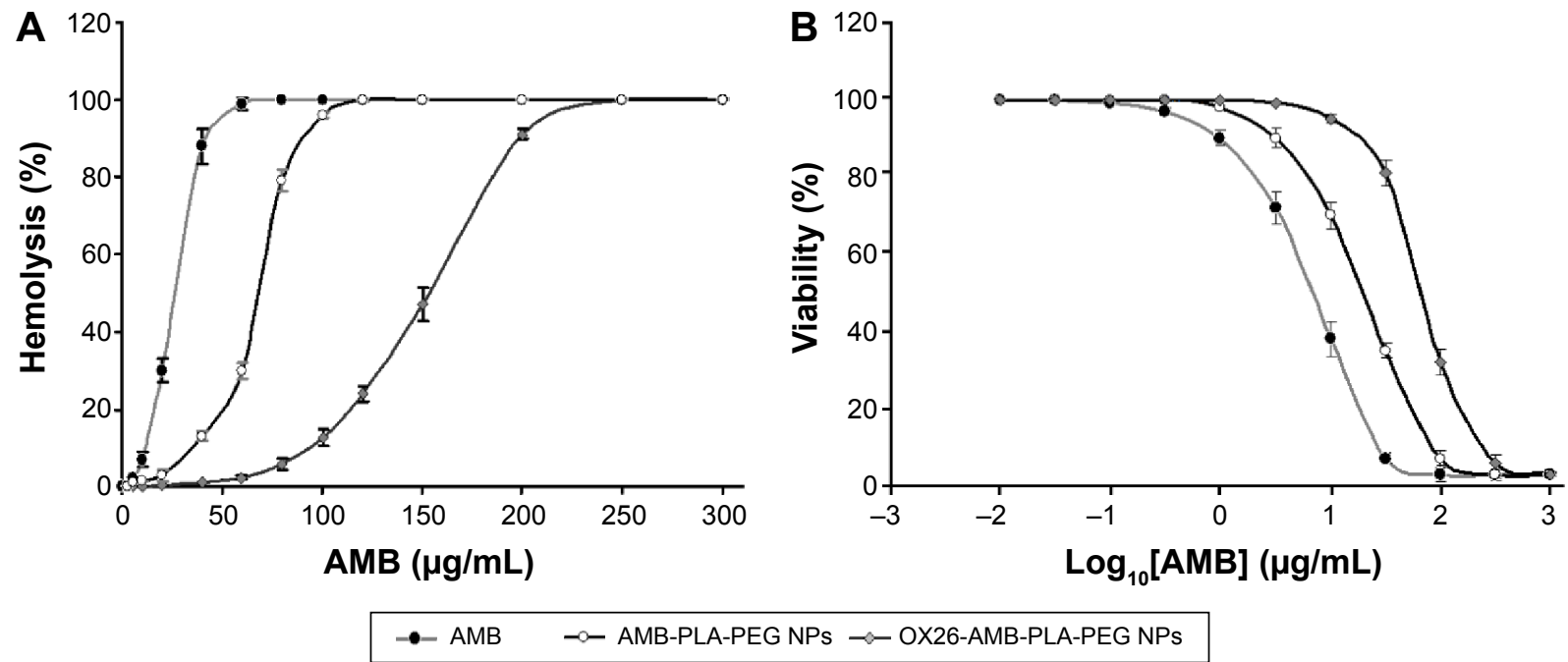

Figure 5 In vitro cytotoxicities of AMB-loaded formulations.

Notes: (A) Hemolysis ratio (\%) of free AMB and AMB-loaded formulations. (B) Cytotoxicity of free AMB and AMB-loaded formulations against CCC-HEK-I cell line with the MTT assay. Data from three independent experiments were expressed as mean \pm SEM $(n=3)$.

Abbreviations: AMB, amphotericin B; NP, nanoparticle; OX26, TfR monoclonal antibody of rats; PBS, phosphate-buffered saline; PEG, polyethylene glycol; PLA, poly(lactic acid); SEM, standard error of mean; TfR, transferrin receptor; MTT, 3-(4,5-dimethylthiazol-2-yl)-2,5-diphenyltetrazolium bromide. 

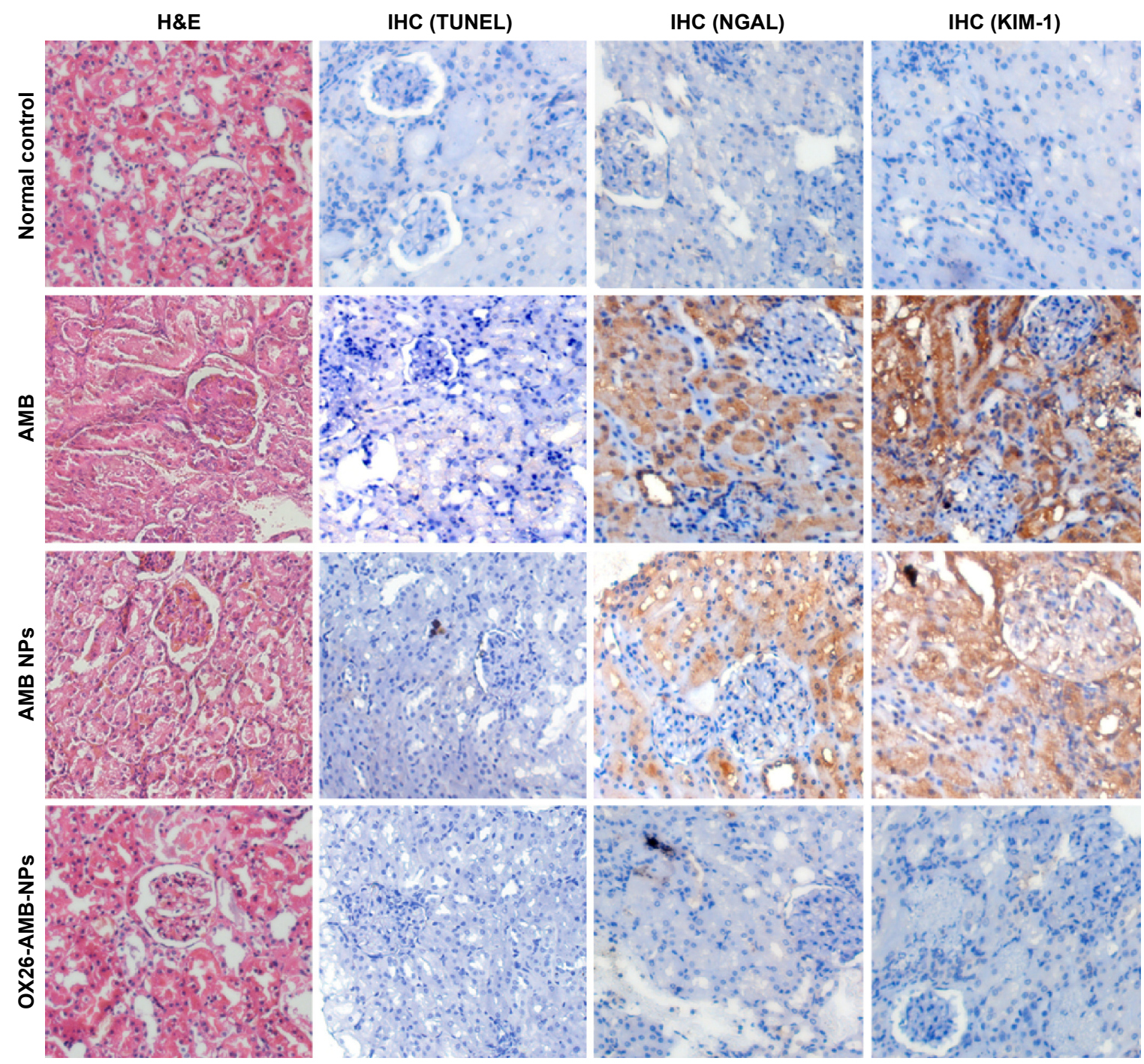

Figure 6 Nephrotoxicity assessment after different AMB formulation treatments.

Notes: Normal control rats show no abnormality and no KIM-I or NGAL expression. AMB and AMB NP treatments show high-level expression of KIM-I and NGAL, but OX26-AMB-NP treatment shows no KIM-I or NGAL expression. All groups of mice show no expression of apoptosis marker TUNEL under AMB therapeutic concentration $(\times 200)$.

Abbreviations: AMB, amphotericin B; H\&E, hematoxylin and eosin stain; IHC, immunohistochemistry; KIM-I, kidney injury molecule-I; NGAL, neutrophil gelatinaseassociated lipocalin; NP, nanoparticle; OX26, TfR monoclonal antibody of rats; TfR, transferrin receptor; TUNEL, terminal deoxynucleotidyl transferase-mediated dUTPbiotin nick end labeling assay.

same high levels of KIM-1 and NGAL, whereas mice treated with OX26-AMB-NP formulations exhibited no congestion, KIM-1, or NGAL expression, suggesting that the OX26-AMB-NPs may cause less nephrotoxicity than deoxycholate AMB.

\section{Drug-induced liver injury}

In order to investigate the hepatotoxicity of different drugs, we measured markers of liver damage and histopathological changes (Figure 7 and Table 2). AMB and AMB-NP treatment caused severe necrosis in hepatic tissues. The degenerative regions of the liver contained necrotic debris and degenerative hepatocytes. However, OX26-AMB-NPs did not cause obvious toxicity to the liver (Figure 7) or lung, heart, or brain (Figure 8).

\section{Cellular uptake}

Cellular uptake of antibody-conjugated PLA-PEG NPs was measured in SKOV3, HeLa (TfR ${ }^{\text {high }}$ ), and HEK 293 (TfR ${ }^{\text {low }}$ ) cell lines (Figure 9A). Cellular uptake of the NPs was much higher on the surface of SKOV3 and HeLa (TfR $\left.{ }^{\text {high }}\right)$ cells than on that of HEK 293 (TfR ${ }^{\text {low}}$ ) cells. SKOV3 and HeLa's surface TfR decreased significantly after mixing with OX26 (Figure 9B and C). These results suggested that the antibody 

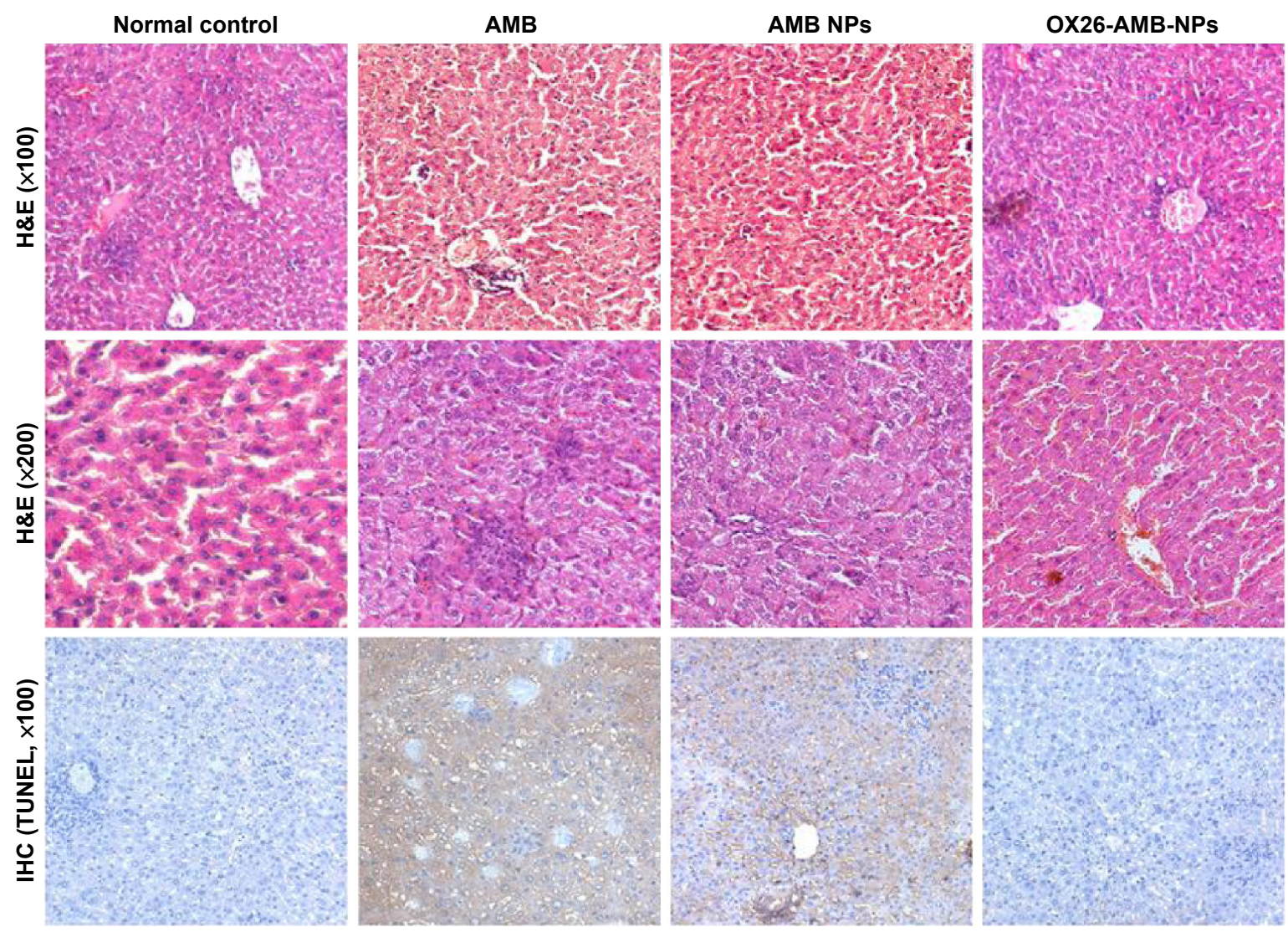

Figure 7 Using TUNEL assay in rats, hepatic histopathology and immunohistochemistry were used to detect cell inflammation and cell apoptosis.

Notes: PBS-treated normal control, showing normal lobular architecture and cell structure; AMB-treated group, showing extensive hepatic centrilobular damage characterized by the presence of vacuolization and severe apoptosis; AMB NPs-treated group, showing modest hepatic centrilobular degeneration and apoptosis; OX26-AMB-NPs, showing lobular architecture and cell structure similar to the normal control.

Abbreviations: AMB, amphotericin B; H\&E, hematoxylin and eosin stain; IHC, immunohistochemistry; NP, nanoparticle; OX26, TfR monoclonal antibody of rats; PBS, phosphate-buffered saline; TfR, transferrin receptor; TUNEL, terminal deoxynucleotidyl transferase-mediated dUTP-biotin nick end labeling assay.

OX26-modified PLA-PEG NPs are actively targeting cells with high TfR expression.

\section{Drug brain distribution}

The concentration of AMB in the serum and brain tissues of mice, dosed two times per day for 3 days with AMB $(3.0 \mathrm{mg} / \mathrm{kg} /$ day $)$, were measured during the following 72 hours (Figure 2A). The concentration of AMB in the serum from

Table 2 Effects of different drug-induced release of ALT, GLDH, and GST $\alpha$

\begin{tabular}{llll}
\hline Group & ALT (U/L) & GLDH (U/L) & GST $\alpha$ \\
\hline Normal control & $31.72 \pm 6.79$ & $11.22 \pm 1.97$ & $58.90 \pm 10.02$ \\
AMB & $143.49 \pm 15.21^{*}$ & $63.78 \pm 5.18^{*}$ & $166.25 \pm 27.29^{*}$ \\
AMB NPs & $58.41 \pm 7.14^{* *}$ & $36.13 \pm 3.95^{* *}$ & $102.83 \pm 14.21^{*}$ \\
OX26-AMB-NPs & $33.17 \pm 6.32$ & $13.05 \pm 3.17$ & $63.07 \pm 7.23$ \\
\hline
\end{tabular}

Notes: Data are presented as mean \pm SEM $(n=10) . * P<0.01, * * P<0.05$, compared with normal control group.

Abbreviations: AMB, amphotericin B; ALT, alanine aminotransferase; GLDH, glutamate dehydrogenase; GST, glutathione-S-transferase; NP, nanoparticle; OX26, TfR monoclonal antibody of rats; SEM, standard error of mean; TfR, transferrin receptor.
12 to 72 hours after the first administration of $3.0 \mathrm{mg} / \mathrm{kg}$ OX26-AMB-NPs was higher than that after the same dose of free AMB or AMB NPs. The serum levels of AMB in mice administered free AMB and AMB NPs did not differ significantly.

The concentration of AMB in the brain tissue began to increase 12 hours after intravenous administration of OX26AMB-NPs, while in mice administered free AMB or AMB $\mathrm{NPs}$, only very low levels of AMB could be detected in the brains. The concentration of AMB in the brains of mice administered free AMB was much lower than the necessary therapeutic dose after 72 hours. In contrast, brain uptake of OX26-AMB-NPs increased quickly and dramatically. The highest concentration of AMB in the brain tissue was detected 60 hours after administration of OX26-AMB-NPs, and the highest brain levels of OX26-AMB-NPs were 5.2-fold higher than the highest concentration detected in animals administered AMB NPs and 6.7-fold higher than that in animals administered free AMB (Figure 2B). The ratio of brain to 


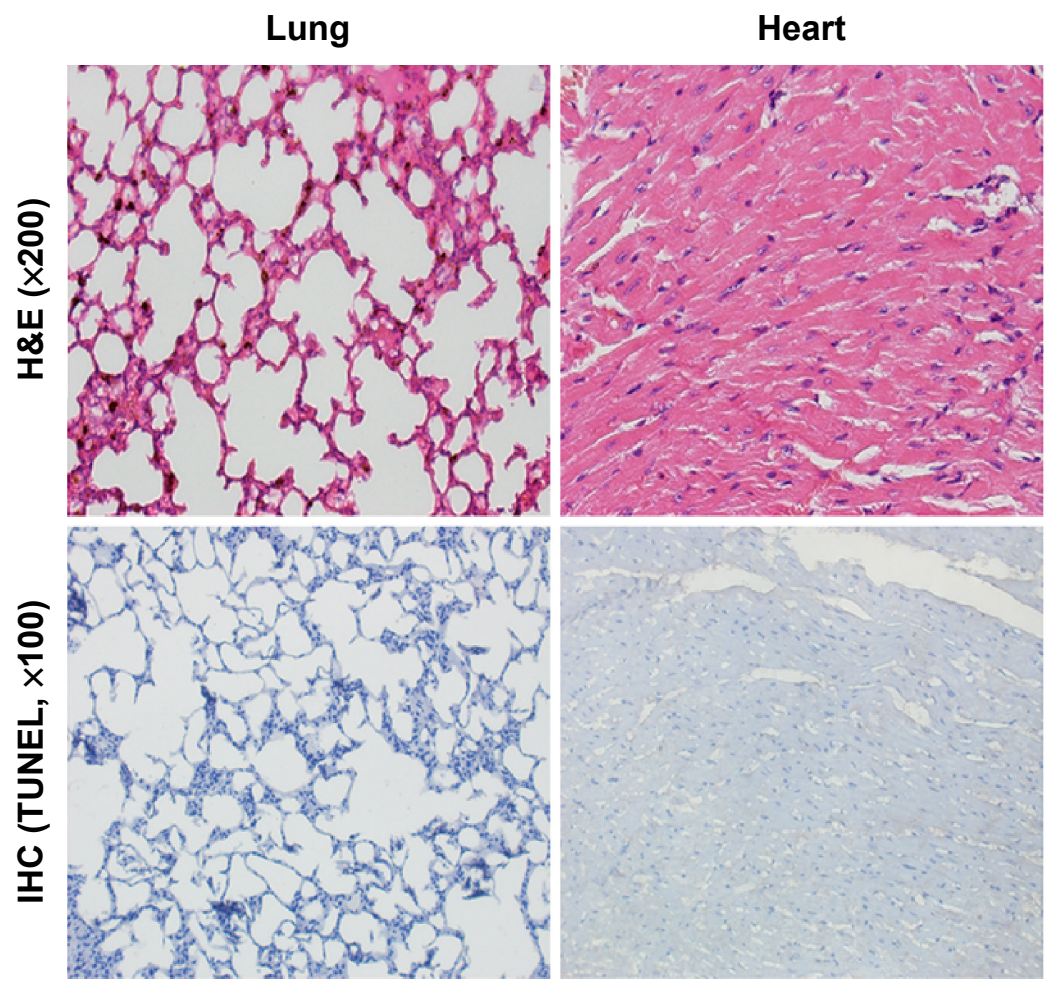

Brain

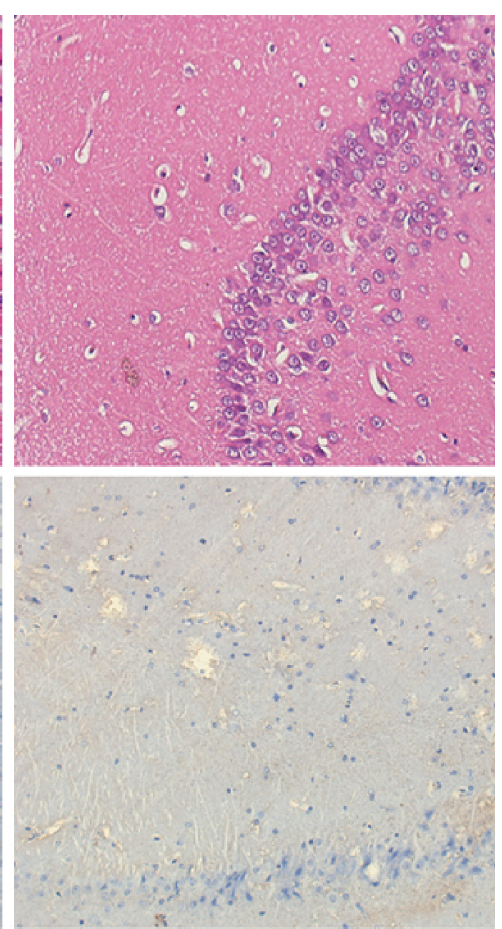

Figure 8 Toxicity assessment of the main tissues after different AMB formulation treatments.

Notes: Macroscopic appearance of histological H\&E and apoptosis marker TUNEL immunohistochemical stains. Histopathological examination of the mice lung/heart/brain detected no obvious pathological changes or cell apoptosis after OX26-AMB-NP (equivalent free AMB, I mg/kg) treatment for 48 hours.

Abbreviations: AMB, amphotericin B; H\&E, hematoxylin and eosin stain; IHC, immunohistochemistry; NP, nanoparticle; OX26, TfR monoclonal antibody of rats; TfR, transferrin receptor; TUNEL, terminal deoxynucleotidyl transferase-mediated dUTP-biotin nick end labeling assay.

serum drug concentration, which represents the efficiency of AMB targeting into the brain tissue, was progressively increased, and then decreased slowly in the OX26-AMB-NPs group, demonstrating the enhanced penetration of OX26AMB-NPs into the brain tissues.

\section{Histopathologic examination}

After 15 days of infection, the histopathology of brain was studied (Figure 10). In model group animals, we detected glabrata spores distributed throughout the brain tissue, many abscesses, and necrosis of the brain parenchyma. The amount of $C$. glabrata spores in the brain parenchyma of animals administered OX26-AMB-NPs was lower, indicating that OX26-AMB-NPs effectively reduced the histological severity of C. glabrata infection.

\section{Survival monitoring}

The lethality rate of mice in the untreated group was $100 \%$ after day 16 postinfection (Figure 11). The OX26-AMB-NP treatment regimen significantly prolonged survival time when compared to the free AMB and the AMB NP regimen $(P<0.01)$. However, there was no significant difference between survivals in mice administered AMB and AMB
$\mathrm{NP}$, consistent with the observed fungal burden observed in the brain.

\section{Discussion}

The BBB is composed of tightly packed endothelial cells supported by surrounding glial cells that aid in the induction and maintenance of a tight junction barrier. These cells are rich in TfR. ${ }^{27,28} \mathrm{AMB}$ is an insoluble broad spectrum antifungal drug that does not efficiently traverse the BBB, making it difficult to achieve effective inhibitory concentrations of AMB in the brain. ${ }^{29}$ High circulating concentrations of AMB can cause hemolysis or severe side effects such as nephrotoxicity and hepatotoxicity. ${ }^{29,30}$ In this study, we designed an AMB formulation combining receptor targeting as a new drug nanocarrier, OX26-AMB-NPs. We then evaluated the physical and biological characteristics of OX26-AMB-NPs.

The results show that the drug-loaded NPs had the ideal size (121.8 \pm 9.9$)$, zeta potential $(-18.1 \pm 1.3 \mathrm{mV})$, and encapsulation efficiency $(84.8 \% \pm 5.3 \%)$. The NPs also exhibited more efficient and sustained drug release, which is conducive to the minimization of adverse effects. Further, at the cytological level, we analyzed the specific adsorption of OX26 


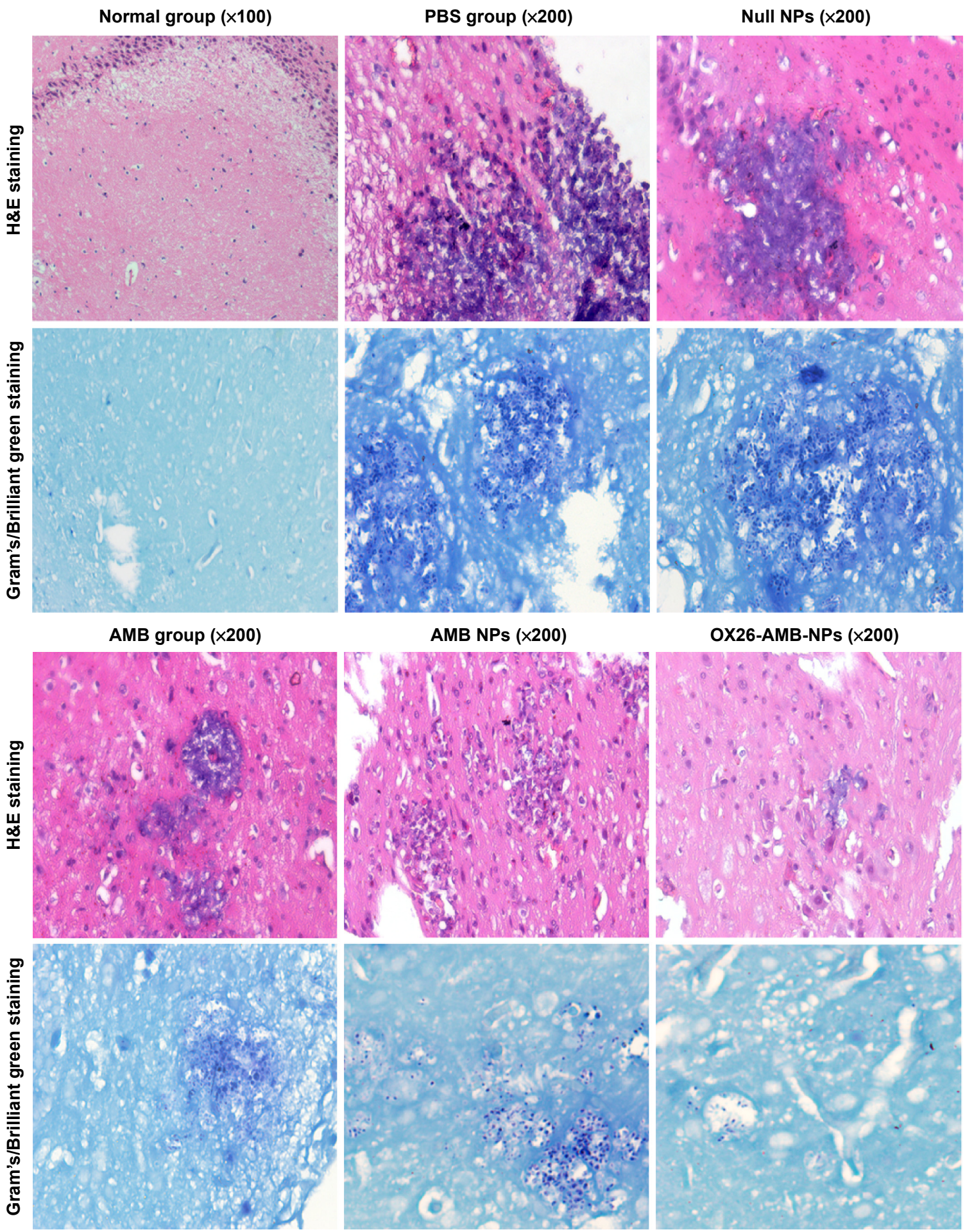

OX26-AMB-NPs $(\times 200)$
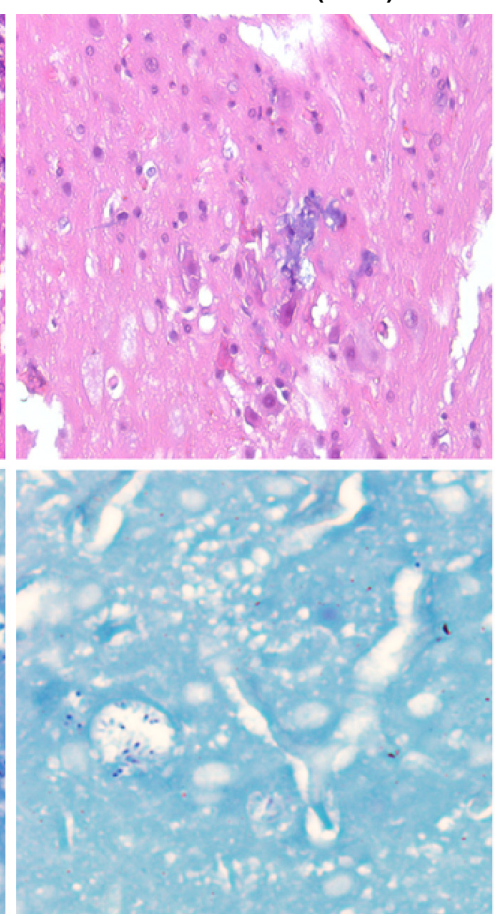

Figure 10 Photomicrographs of brain section showing the treatment efficacy of AMB formulations for Candida glabrata infection on day I5 after inoculation.

Notes: A histopathological analysis of H\&E staining and Gram's/Brilliant green staining for brain sections were performed. Groups: normal group, PBS group, null NPs group, AMB group, AMB NPs group, and OX26-AMB-NPs group.

Abbreviations: AMB, amphotericin B; H\&E, hematoxylin and eosin stain; NP, nanoparticle; OX26, TfR monoclonal antibody of rats; PBS, phosphate-buffered saline; TfR, transferrin receptor.

NPs to $\mathrm{TfR}^{+}$cells, internalization, and uptake efficiency, and the results confirmed that $\mathrm{TfR}^{+}$cells can specifically internalize OX26-AMB-NPs, suggesting that OX26-AMBNPs have a specific biological effect on TfRs.
The successfully developed CNS-targeting PLA-PEG NP micellar formulation of AMB, modified by addition of OX26, can improve brain permeability through TfRmediated transcytosis. In this work, we have demonstrated 


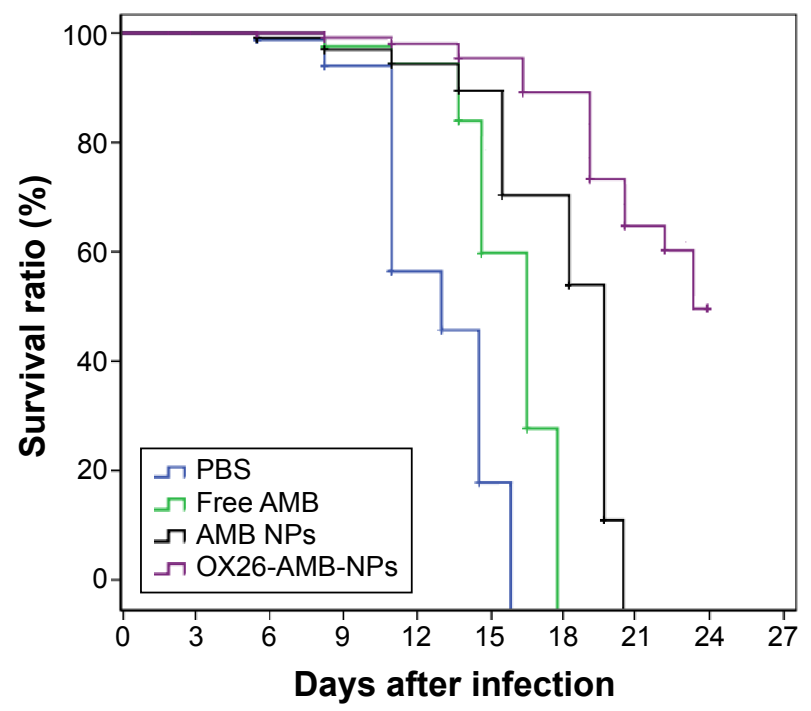

Figure II Survival curves of BALB/c mice intracranially infected with Candida glabrata $10^{5} \mathrm{CFU} /$ animal and treated with free AMB, AMB NPs, and OX26-AMB$\mathrm{NPs}$ at $3 \mathrm{mg} / \mathrm{kg}$ from day 2 to 4 after infection.

Abbreviations: AMB, amphotericin B; CFU, colony-forming unit; NP, nanoparticle; OX26, TfR monoclonal antibody of rats; PBS, phosphate-buffered saline; TfR, transferrin receptor.

that OX26-AMB-NPs can rapidly and efficiently deliver AMB into brain tissues of mice, significantly enhancing the therapeutic efficacy of $\mathrm{AMB}$ and efficiently reducing CNS fungal infection. OX26-AMB-NPs achieved better efficacy than AMB-NPs without OX26 and free AMB formulations. Treatment of the fungal infection of the CNS reflects the efficiency of delivery of the drug into the brain tissue.

In addition, we also evaluated the toxicity and adverse effects associated with administration of OX26-AMB-NPs in a mouse model. The nanomaterial packaging strategy effectively prevented AMB-mediated hemolysis and renal toxicity, mainly attributed to the improved solubility of AMB by PEG, thus increasing the bioavailability and the sustained release of AMB, which retained a stable blood drug concentration with higher efficacy and an extended therapeutic window. The most significant finding was that the OX26-AMB-NPs retained high stability in the blood, and blood drug concentration decay was slow.

We used HLPC to analyze the concentration distribution curves of AMB in the blood and brain tissues of mice. We observed that OX26-AMB-NPs achieved significantly improved concentration distribution in the CNS. At therapeutic doses, AMB quickly reaches the CNS, unlike administration of free AMB or AMB NPs. OX26-AMB-NPs can deliver $\mathrm{AMB}$ into the $\mathrm{CNS}$ and promotes brain parenchyma accumulation, reducing fungal colony formation in brain tissue and improving survival time.

Intravenous injection of OX26-AMB-NPs significantly improved the efficacy of AMB, reduced the fungal burden in brain tissue and the severity of histopathological changes, and prolonged survival time. The research indicates that the OX26-AMB-NPs brain targeting delivery system can improve penetration into the CNS and enhance the effective therapeutic level of drug in the brain. We conclude that OX26 promotes polymeric micelles to bond with cells of the BBB, inducing internalization, and thus improving the transport capacity and accumulation in the CNS. Therefore, the OX26-modified brain-targeting delivery system could have promising applications in the treatment of intracerebral fungal infection.

\section{Acknowledgments}

This study was supported by the National High Technology Research and Development Program (863 Program) (grant number 2011AA02A111) and the Open Research Fund Program of the State Key Laboratory of Virology of the People's Republic of China (grant number 2014KF004), the National Natural Science Fund (81572431), and the Anhui College Students' Innovation and Entrepreneurship Training Plan (AH201410361087, AH201410361263, AH201410361264, AH201410361266, AH201410361269, 20151036317).

\section{Disclosure}

The authors report no conflicts of interest in this work.

\section{References}

1. Yu Y, Du L, Yuan T, et al. Risk factors and clinical analysis for invasive fungal infection in neonatal intensive care unit patients. Am J Perinatol. 2013;30(7):589-594.

2. Post MJ, Thurnher MM, Clifford DB, et al. CNS-immune reconstitution inflammatory syndrome in the setting of HIV infection, part 2: discussion of neuro-immune reconstitution inflammatory syndrome with and without other pathogens. AJNR Am J Neuroradiol. 2013;34(7):1308-1318.

3. Badiee P, Kordbacheh P, Alborzi A, Zeini F, Mirhendy H, Mahmoody M. Fungal infections in solid organ recipients. Exp Clin Transplant. 2005;3(2): 385-389.

4. Pettit AC, Kropski JA, Castilho JL, et al. The index case for the fungal meningitis outbreak in the United States. N Engl J Med. 2012;367(22): 2119-2125.

5. Shao K, Wu J, Chen Z, et al. A brain-vectored angiopep-2 based polymeric micelles for the treatment of intracranial fungal infection. Biomaterials. 2012;33(28):6898-6907.

6. Tribble DR, Rodriguez CJ. Combat-related invasive fungal wound infections. Curr Fungal Infect Rep. 2014;8(4):277-286.

7. Azik FM, Tezer H, Parlakay AO, et al. Secondary antifungal prophylaxis in pediatric hematopoietic stem cell transplants. J Pediatr Hematol Oncol. 2015;37(1):e19-e22.

8. Mohamadi J, Motaghi M, Panahi J, et al. Anti-fungal resistance in candida isolated from oral and diaper rash candidiasis in neonates. Bioinformation. 2014;10(11):667-670.

9. Antunes AM, Teixeira C, Corvo ML, Perdigoto R, Barroso E, Marcelino P. Prophylactic use of liposomal amphotericin B in preventing fungal infections early after liver transplantation: a retrospective, single-center study. Transplant Proc. 2014;46(10):3554-3559.

10. Kassab R, Parrot-Lopez H, Fessi H, Menaucourt J, Bonaly R, Coulon J. Molecular recognition by Kluyveromyces of amphotericin B-loaded, galactose-tagged, poly(lactic acid) microspheres. Bioorg Med Chem. 2002;10(6):1767-1775. 
11. Zhou W, Wang Y, Jian J, Song S. Self-aggregated nanoparticles based on amphiphilic poly(lactic acid)-grafted-chitosan copolymer for ocular delivery of amphotericin B. Int J Nanomedicine. 2013;8:3715-3728.

12. Ren $\mathrm{T}, \mathrm{Xu} \mathrm{N}$, Cao C, et al. Preparation and therapeutic efficacy of polysorbate-80-coated amphotericin B/PLA-b-PEG nanoparticles. J Biomater Sci Polym Ed. 2009;20(10):1369-1380.

13. Lipa S, Sunita Y, Mansoor A. Nanotechnology for CNS delivery of bio-therapeutic agents. Drug Deliv Transl Res. 2013;3(4):336-351.

14. Kim K, Yu M, Zong X, et al. Control of degradation rate and hydrophilicity in electrospun non-woven poly(D, L-lactide) nanofiber scaffolds for biomedical applications. Biomaterials. 2003;24(27):4977-4985.

15. Gabizon A, Horowitz AT, Goren D, et al. Targeting folate receptor with folate linked to extremities of poly(ethylene glycol)-grafted liposomes: in vitro studies. Bioconjug Chem. 1999;10(2):289-298.

16. Elvin Blanco, Erik A Bey, Ying Dong, et al. $\beta$-Lapachone-containing PEG-PLA polymer micelles as novel nanotherapeutics against NQO1overexpressing tumor cells. J Control Release. 2007;122(3):365-374.

17. Karim SS, Mahmoud ES, Luca C, et al. Determination of factors controlling the particle size and entrapment efficiency of noscapine in PEG/ PLA nanoparticles using artificial neural networks. Int J Nanomedicine. 2014;9:4953-4964.

18. Tang X, Dai H, Zhu Y, et al. Maytansine-loaded star-shaped folate-core PLA-TPGS nanoparticles enhancing anticancer activity. Am J Transl Res. 2014;6(5):528-537.

19. Sade H, Baumgartner C, Hugenmatter A, Moessner E, Freskgård PO, Niewoehner J. A human blood-brain barrier transcytosis assay reveals antibody transcytosis influenced by $\mathrm{pH}$-dependent receptor binding. PLoS One. 2014;9(4):e96340.

20. Bansal V, Kumar M, Dalela M, Brahmne HG, Singh H. Evaluation of synergistic effect of biodegradable polymeric nanoparticles and aluminum based adjuvant for improving vaccine efficacy. Int J Pharm. 2014;471(1-2):377-384.

21. Yamasaki M, Harada E, Tamura $Y$, et al. In vitro and in vivo safety and efficacy studies of amphotericin B on Babesia gibsoni. Vet Parasitol. 2014;205(3-4):424-433.
22. Shimada C, Uesawa Y, Ishihara M, et al. Quantitative structure-cytotoxicity relationship of piperic acid amides. Anticancer Res. 2014;34(9): 4877-4884.

23. Capilla J, Flavia A, Mayayo E, Guarro J. Efficacy of intrathecal liposomal amphotericin B plus oral posaconazole in the treatment of acute meningeal cryptococcosis in a murine model. Int J Antimicrob Agents. 2013;42(3):282-283.

24. O’Connor L, Livermore J, Sharp AD, et al. Pharmacodynamics of liposomal amphotericin B and flucytosine for cryptococcal meningoencephalitis: safe and effective regimens for immunocompromised patients. J Infect Dis. 2013;208(2):351-361.

25. Urbschat A, Obermüller N, Paulus P, et al. Upper and lower urinary tract infections can be detected early but not be discriminated by urinary NGAL in adults. Int Urol Nephrol. 2014;46(12):2243-2249.

26. Martins SM, Sarmento B, Nunes C, Lúcio M, Reis S, Ferreira DC. Brain targeting effect of camptothecin-loaded solid lipid nanoparticles in rat after intravenous administration. Eur J Pharm Biopharm. 2013;85 (3 Pt A):488-502.

27. Booth R, Kim H. Permeability analysis of neuroactive drugs through a dynamic microfluidic in vitro blood-brain barrier model. Ann Biomed Eng. 2014;42(12):2379-2391.

28. Molino Y, Jabès F, Lacassagne E, Gaudin N, Khrestchatisky M. Setting-up an in vitro model of rat blood-brain barrier (BBB): a focus on BBB impermeability and receptor-mediated transport. J Vis Exp. 2014; (88):e51278

29. Chu H, Ding H, Tang Y, Dong Q. Erythropoietin protects against hemorrhagic blood-brain barrier disruption through the effects of aquaporin-4 Lab Invest. 2014;94(9):1042-1053.

30. Petraitiene R, Petraitis V, Lyman CA, et al. Efficacy, safety, and plasma pharmacokinetics of escalating dosages of intravenously administered ravuconazole lysine phosphoester for treatment of experimental pulmonary aspergillosis in persistently neutropenic rabbits. Antimicrob Agents Chemother. 2004;48(4):1188-1196.
International Journal of Nanomedicine

\section{Publish your work in this journal}

The International Journal of Nanomedicine is an international, peerreviewed journal focusing on the application of nanotechnology in diagnostics, therapeutics, and drug delivery systems throughout the biomedical field. This journal is indexed on PubMed Central,

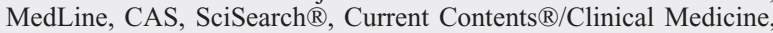

\section{Dovepress}

Journal Citation Reports/Science Edition, EMBase, Scopus and the Elsevier Bibliographic databases. The manuscript management system is completely online and includes a very quick and fair peer-review system, which is all easy to use. Visit http://www.dovepress.com/ testimonials.php to read real quotes from published authors. 